\title{
sigo \\ Estado actual de las investigaciones de la cerámica indígena del norte de Mendoza. Tecnología, cronología y distribución
} RMA

\author{
North Mendoza Indigenous pottery analysis current \\ state. Technology, chronology and distribution
}

VI Jornadas

Arqueológicas Cuyanas

\author{
Cristina Prieto Olavarría*, Horacio Chiavazza**, \\ Leonardo Castillo***, Vanina Tobar****, \\ Enzo Bontorno****, Vanina Porta******
}

\begin{abstract}
* CONICET, Instituto Argentino de Nivología Glaciología y Ciencias Ambientales, CCT-MENDOZA. Facultad de Filosofía y Letras, Universidad Nacional de Cuyo. cprieto@mendoza-conicet.gob.ar ** Laboratorio de Arqueología Histórica y Etnohistoria, Facultad de Filosofía y Letras, Universidad Nacional de Cuyo. Centro de Investigaciones Ruinas de San Francisco. hchiavazza@gmail.com *** CONICET, Instituto Argentino de Nivología Glaciología y Ciencias Ambientales, CCT-MENDOZA. Facultad de Filosofía y Letras, Universidad Nacional de Cuyo. Icastillo@mendoza-conicet.gob.ar **** Centro de Investigaciones Ruinas de San Francisco. vantob3@yahoo.com.ar ${ }^{* * * * * *}$ Laboratorio de Arqueología Histórica y Etnohistoria, Facultad de Filosofía y Letras, Universidad Nacional de Cuyo. Centro de Investigaciones Ruinas de San Francisco. vanipor@hotmail.com **** Laboratorio de Arqueología Histórica y Etnohistoria, Facultad de Filosofía y Letras, Universidad Nacional de Cuyo. Centro de Investigaciones Ruinas de San Francisco.enzo_bontorno77@hotmail.com
\end{abstract}

\begin{abstract}
Resumen
Análisis tecno-tipológicos sobre cerámica arqueológica del norte de la provincia de Mendoza permitieron definir la variabilidad en la producción local en un lapso de casi mil años, entre aproximadamente los ca. 1200-1300 años AP. A los tipos cerámicos más conocidos y ampliamente distribuidos, Agrelo y Viluco, se sumaron otros grupos cerámicos: Gris/Marrón, Anarajado Rojizo y Viluco Colonial. Además, los tipos locales conocidos para los tambos incaicos de Uspallata se hallaron en sitios de precordillera y piedemonte alto, y también se encontraron tiestos de cerámica foránea, como los tipos Tocota, Diaguita y Copiapó Negro sobre Rojo en los diversos ambientes del área. Las dataciones absolutas permitieron ajustar las cronologías y a partir del análisis petrográfico se detectó la correspondencia de las inclusiones con la geología local y la presencia de materias primas foráneas, lo que se interpreta como movilidad y/o intercambio con áreas vecinas.
\end{abstract}

Palabras clave: cerámica; Mendoza; tecnología; tipología; cronología; distribución.

\begin{abstract}
Technological and typological analysis of archaeological ceramics of northern Mendoza province allowed to define the variability in local production over a period of almost a thousand years, between ca. 1200-1300 years BP. Agrelo and Viluco are the best known and widely distributed ceramic types and other ceramic groups were defined: Gris/ Marrón, Anarajando Rojizo and the Viluco Colonial. Local types manufactured in Inca Uspallata tambos were found at sites of the high-foothill and the piedmont. Foreign ceramic types also Tocota, Diaguita and Copiapó Negro sobre Rojo, were found isolated. Meanwhile, radiocarbon and termoluminscence dating allowed to clarify the chronologies, and petrographic analysis allowed detect the correspondence of the inclusions with the local geology and in other cases the presence of foreign raw materials, indicating mobility and/or exchanges with neighboring areas.
\end{abstract}

Keywords: ceramics; Mendoza; technology; chronology; distribution.

Los estudios sobre la cerámica arqueológica en el norte y centro de la provincia de Mendoza comenzaron junto a las primeras investigaciones de principios del siglo XX (Boman 1920; Rusconi 1962; entre otros). Entre las décadas de 1950 y 1970 cristalizaron las definiciones de los principales tipos alfareros, Agrelo y Viluco (Canals Frau 1956; Lagiglia 1978). Posteriormente, se precisaron los estudios de cerámicas de diversos sitios arqueológicos y 
se asociaron a los períodos alfareros Temprano, Medio y Tardío (Bárcena y Román 1990; Chiavazza 1995; Durán y García 1990; García 1995, entre otros); se realizaron dataciones absolutas que afinaron la cronología del área (Bárcena 1998); se detalló la secuencia propuesta por Canals Frau (cerámicas grises tempranas y anaranjadas tardías); se propuso que la cerámica Viluco se originó posteriormente al ingreso incaico (García 1996).

Desde el año 2000 los estudios cerámicos se centraron en aspectos tecnológicos, de forma y decoración, y se estudió en profundidad la cerámica Viluco producida entre el período Alfarero Tardío y colonial temprano en el norte mendocino (Cahiza 2001; Prieto Olavarría 2012; Prieto Olavarría y Chiavazza 2009; Prieto Olavarría y Páez 2015; Prieto Olavarría y Chiavazza 2010; Tobar 2013; entre otros). Los estudios en el centro provincial (Valle de Uco), incluyeron el análisis comparativo de Viluco con los tipos producidos en los tambos incaicos del valle de Uspallata (Ots 2008). En término generales, los resultados corroboraron que la cerámica Viluco fue producida desde el ingreso de la dominación incaica en el área (García 1996; Ots 2008; Prieto Olavarría 2012). En el caso de Agrelo y de los conjuntos cerámicos asociados a contextos del período Alfarero Medio, se estudió la cerámica de la planicie NE (Castillo et al. 2007; Chiavazza 2001, 2007, 2012; Chiavazza y Prieto 2008; Prieto Olavarría y Castro de Machuca 2015; Prieto Olavarría et al. 2016) y de la precordillera (Frigolé et al. 2014; Prieto Olavarría y
Castro de Machuca 2015). Por su parte, los trabajos que mencionan a las cerámicas correspondientes al período Alfarero Temprano son menos abundantes (Chiavazza 1995; Prieto Olavarría et al. 2015).

Nuestras investigaciones se enmarcan en una escala regional y permiten aportar una visión amplia de la variabilidad tecnológica y cronológica de la alfarería del norte de Mendoza. El objetivo de este manuscrito es presentar una síntesis de la dispersión, cronología y principales resultados del análisis tecno-tipológico (análisis morfométrico, de huellas de uso, funcional, decorativo, de pasta y de huellas post-depositacionales) de los tipos cerámicos y conjuntos procedentes de 73 puntos arqueológicos (PA) ubicados en ambientes de precordillera, piedemonte y planicie NE de la provincia de Mendoza, faja central de la Subárea Arqueológica Centro Oeste Argentina (Figura 1).

\section{Material y método}

Los fragmentos analizados proceden de excavaciones y de recolecciones superficiales (Tabla 1 y 2). El estudio tecno-tipológico se estandarizó para sistematizar las comparaciones entre los materiales de diferentes unidades ambientales. El análisis macroscópico y submacroscópico (pasta) se basa en diversas propuestas analíticas (Rice 1987, entre otros) y se relevó información tecnológica, morfológica, decorativa, huellas de uso y

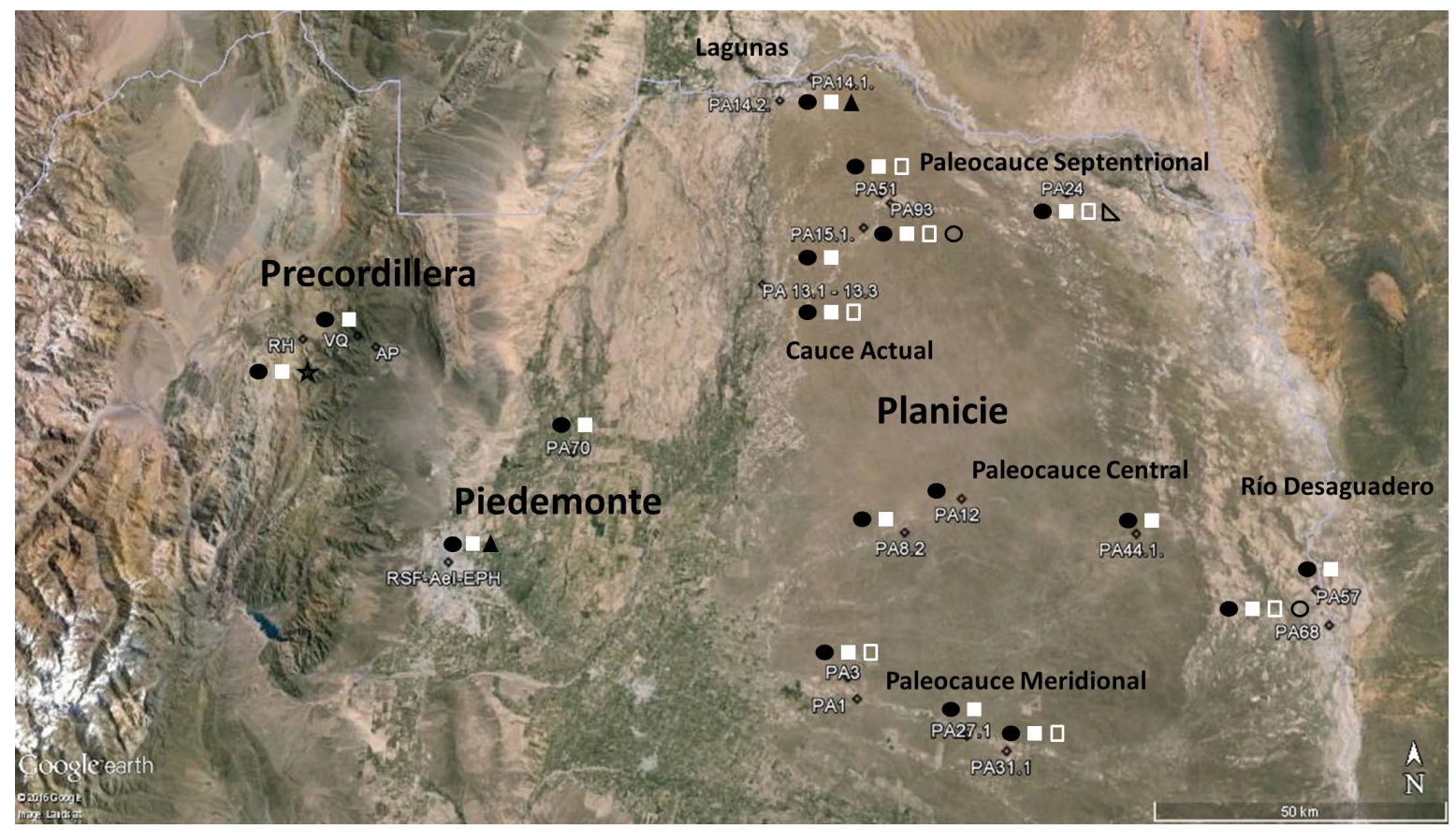

Figura 1. Norte de Mendoza y sus diversos ambientes. Esquemáticamente se muestra la distribución de las diversos tipos y conjuntos cerámicos: cuadrado blanco lleno: tipo Viluco; cuadrado blanco vacío: cerámica Anaranjada Rojiza; círculo negro lleno: tipo Agrelo; círculo negro vacío: cerámica Gris/Marrón; estrella negra vacía: tipo Tocota; triángulo negro lleno: cerámica Diaguita; triángulo rectángulo vacío: tipo Copiapó Negro sobre Rojo.

Figure 1. North Mendoza and its diverse environments. Schematically it is shown the distribution of various ceramic types and assemblies: cuadrado blanco lleno: Viluco type; cuadrado blanco vacío: Anaranjada Rojiza pottery; círculo negro lleno: Agrelo type; círculo negro vacío: Gris/Marrón pottery; estrella negra vacía: Tocota type; triángulo negro lleno: Diaguita pottery; triángulo rectángulo vacío: Copiapó Negro sobre Rojo type. 


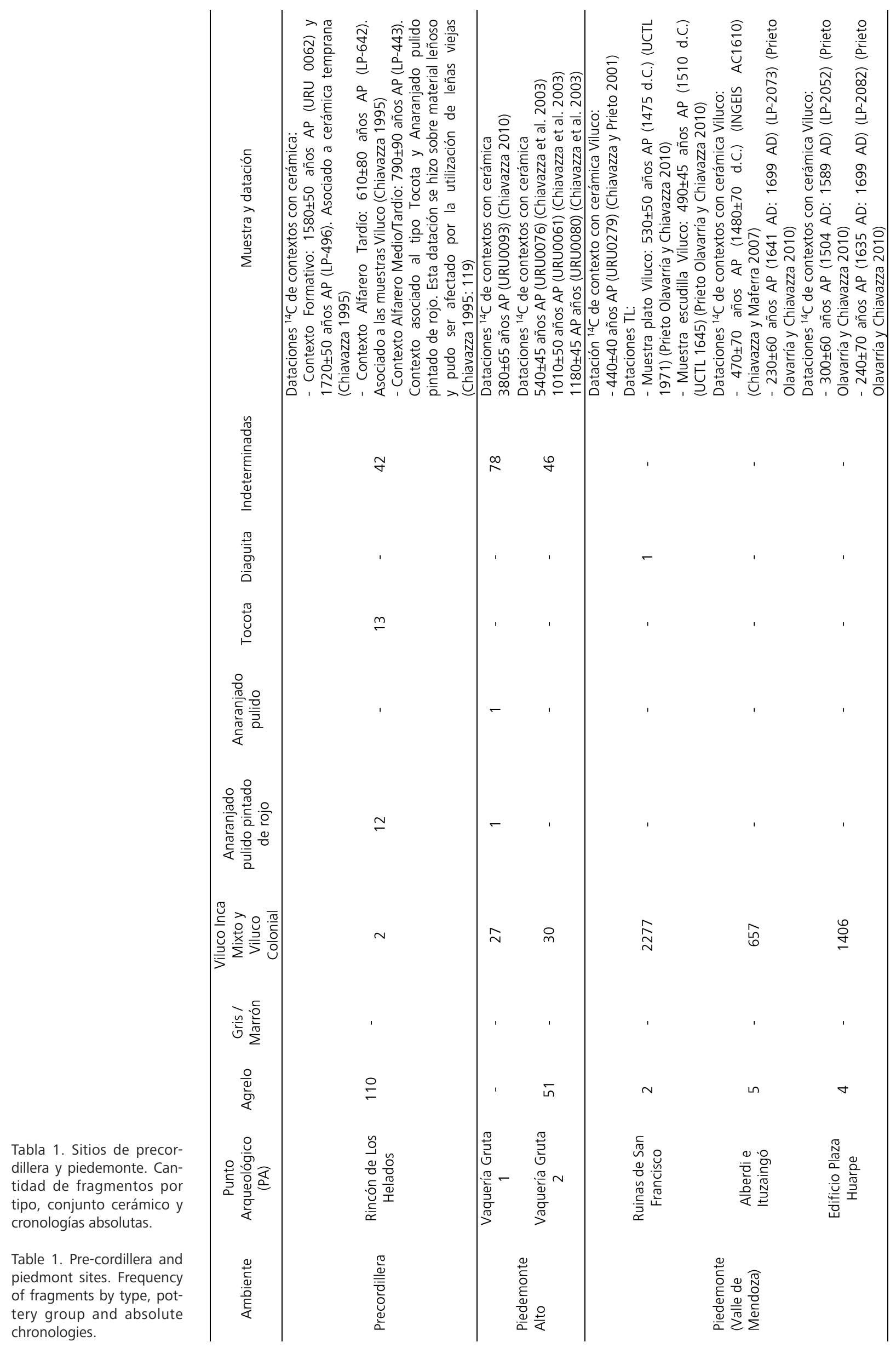




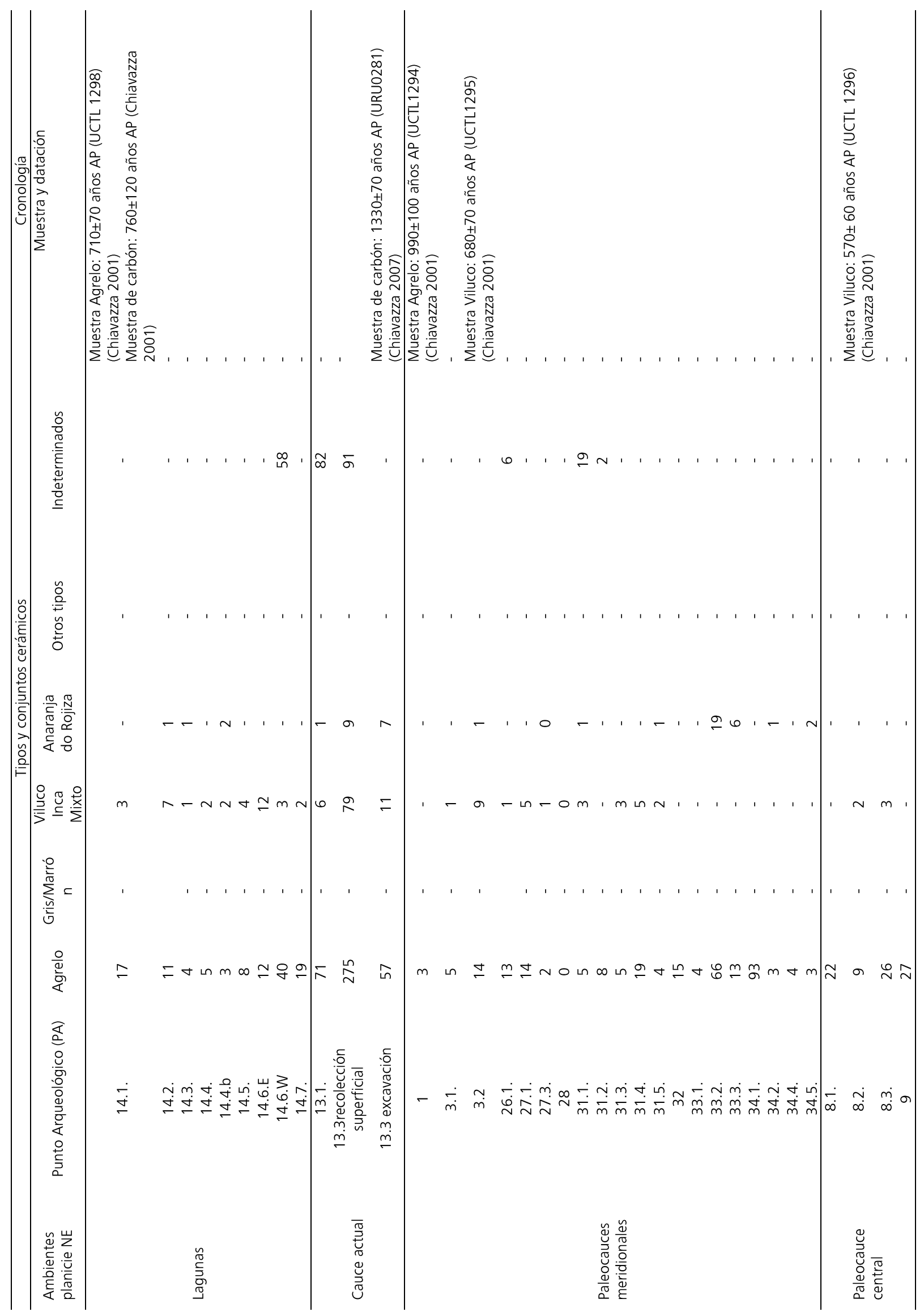

Tabla 2. Sitios de la Planicie NE. Cantidad de fragmentos por tipo, conjuntos cerámicos y cronologías absolutas. (Continúa en próxima página) Table 2. Northeastern plains sites. Frequency of fragments by type, pottery group and absolute chronologies. (Continues in next page) 


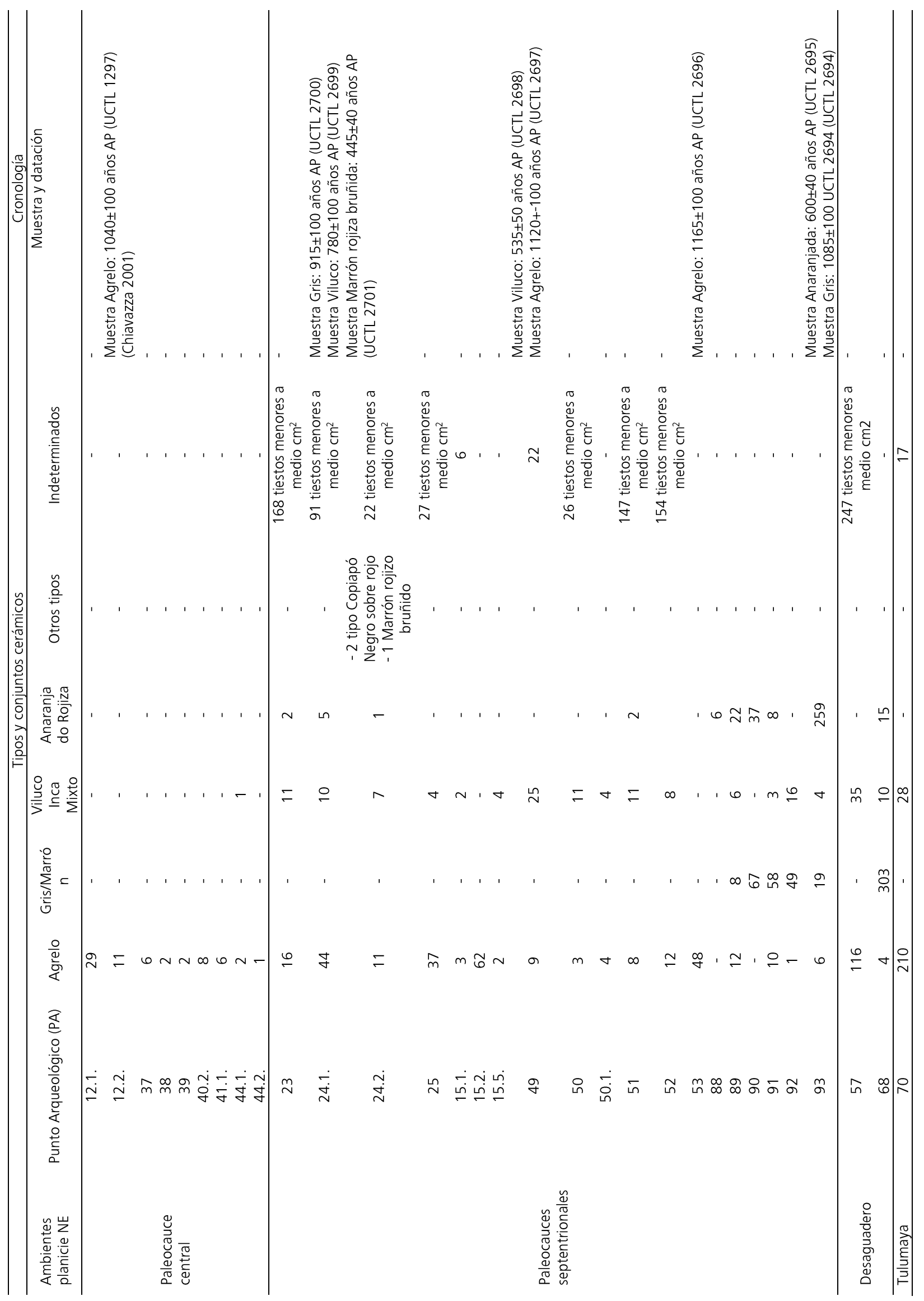

Tabla 2. Sitios de la Planicie NE. Cantidad de fragmentos por tipo, conjuntos cerámicos y cronologías absolutas. (Continuación de página previa) Table 2. Northeastern plains sites. Frequency of fragments by type, pottery group and absolute chronologies. (Continues from previous page) 
huellas post-depositacionales (Prieto Olavarría 2012; Prieto y Chiavazza 2009; Prieto Olavarría y Páez 2015; Prieto Olavarría et al. 2016). Con base en el estudio con lupa binocular (10X - 40X) de los cortes frescos de las pastas, se definieron patrones y familias de pasta para cada tipo, y se confeccionó un patrón general comparativo de pastas para todo el norte de Mendoza (Castillo 2007, Chiavazza 2007, Prieto Olavarría 2010a, Prieto Olavarría et al. 2016). También se hizo el análisis petrográfico de las láminas delgadas de gran parte de los patrones de pasta (lupa binocular) con el objetivo de conocer los atributos composicionales de las inclusiones (origen geológico de rocas, minerales y microfósiles) y de la textura en general. Estos se basaron en la metodología propuesta por Whitbread (1996), que postula caracterizar grupos de fábrica de acuerdo al origen geológico de las inclusiones más conspicuas y los atributos de microestructura.

\section{Resultados}

Los estudios realizados permitieron conocer en mayor detalle los atributos tecnológicos, la dispersión y la cronología de los tipos y conjuntos cerámicos del norte de Mendoza. La cerámica Agrelo es el tipo tradicionalmente descripto para el período Alfarero Medio y fue caracterizado a partir de las formas y decoraciones desde mediados del siglo XX (Canals Frau 1956; Michieli 1974). Estos primeros trabajos se centraron en las vasijas recuperadas en la zona nucleares definida para la cultura Agrelo (distrito de Agrelo, departamento de Luján de Cuyo) y de otros sitios ubicados en los departamentos de San Martín y Tupungato, y son la base a partir de la cual se analiza la alfarería de este período. Agrelo se definió como un conjunto representado por vasijas medianas y grandes con cuellos altos, tazones o pucos con dos asas, las superficies grises, la cocción reductora y las bases con huellas de cestería (Canals Frau 1956, Michieli 1974, García 1995). La decoración se clasificó en: estriada; incisa (chevrones o escalerados en cuello o cuerpo de vasijas con cuello, tazones o pucos); imbricada o corrugada (cuerpo de las vasijas: cuello y pucos con dos asas); modelada e incisa (mamelones con incisiones punteadas, bandas incisas y motivos antropomorfos) (Canals Frau y Semper 1956). Los resultados petrográficos obtenidos de tiestos recuperados en diversos sitios de la planicie NE, permitieron identificar dos grupos de fábrica (Sedimentario volcánico; Sedimentario caliza volcánico metamórfico). En ambos, destaca la predominancia de rocas de origen sedimentario y que las inclusiones se corresponden con la geología del norte de Mendoza (Prieto Olavarría y Castro de Machuca 2015).

Los estudios de la cerámica del período Alfarero Medio procedente de los sitios de la planicie $\mathrm{NE}$, reveló variabilidad en los conjuntos y la dificultad de adscribirla tipológicamente a Agrelo (Figura 2 A, B, C), por lo cual se optó por denominar cerámica del "Medio" a aquellos conjuntos que no se correspondían completamente a
Agrelo (Prieto Olavarría y Castro de Machuca 2015). Esta idea de variabilidad también se fundamentó en los resultados de los análisis petrográficos, ya que un patrón de pasta adjudicado preliminarmente al tipo Agrelo, presentó pegmatitas (grupo de fábrica Granítico pegmatitas, Figura 2 C), un tipo de roca que no está presente en la geología del norte de Mendoza, pero si en las Sierras Centrales. Esta evidencia permitió postular la movilidad y/o intercambio de las poblaciones del norte mendocino con aquellas ubicadas en áreas vecinas al este y noreste (Prieto Olavarría y Castro de Machuca 2015). Al respecto, los resultados obtenidos del análisis de tecnología lítica de los mismos sitios, también evidenciaron la presencia de materias primas de Sierras Centrales (Chiavazza 2001).

La cerámica del Medio, se caracteriza por conjuntos fragmentarios con superficies de tonalidades grises, negras o marrones, alisadas, algunas con decoración incisa o estriada y atmósfera de cocción reductora u oxidante (Figura 2 B). Petrográficamente se han detectado siete grupos de fábrica (Sedimentario volcánico; Sedimentario caliza volcánico metamórfico; Sedimentario volcánico inclusiones arcillosas; Caliza volcánico sedimentario; Volcánico; Granítico; Granítico pegmatitas), cuatro de los cuales son particulares de este grupo, uno está presente en el tipo Agrelo y todos se corresponden con el perfil geológico local (Prieto Olavarría y Castro de Machuca 2015). Considerando que este grupo está en proceso de estudio, proponemos ampliar su denominación con el término cerámica Gris/Marrón (Figura 2 B).

La cerámica Agrelo tiene una amplia dispersión (Figura 1), ya que trasciende los sitios precordilleranos y del piedemonte tradicionalmente conocidos (Canals Frau 1956; Bárcena 1998, entre otros), y se distribuye hacia el este y noreste hasta el río Desaguadero (Tabla 2, Figura 1). Por su parte, la cerámica Gris/Marrón se encuentra en sitios de la planicie NE (Tabla 2, Figura 1). Las dataciones absolutas obtenidas para ambos conjuntos recuperados en la planicie NE se ubican mayoritariamente en el rango entre los 900 y 1200 años $A P$, si bien se obtuvo una datación más tardía para una muestra de Lagunas (710 \pm 70 años AP, UCTL 1298) (Tabla 2).

En las recientes investigaciones en el PA68, ubicado en el margen oeste del río Desaguadero, detectamos algunos fragmentos Agrelo y gran cantidad del conjunto Gris/ Marrón (Figura 1). En este último caso, los patrones de pasta se corresponden o son similares a los que tienen inclusiones de las Sierras Centrales (Prieto Olavarría et al. 2016). Esta información deberá ser confirmada con análisis petrográficos y los resultados permitirán evaluar la movilidad poblacional y/o el intercambio en esta área considerada tanto el límite cultural de los desarrollos del norte mendocino, como un corredor de circulación con eje norte-sur, por lo cual habría concentrado el movimiento de grupos portadores de diferentes tradiciones alfareras 


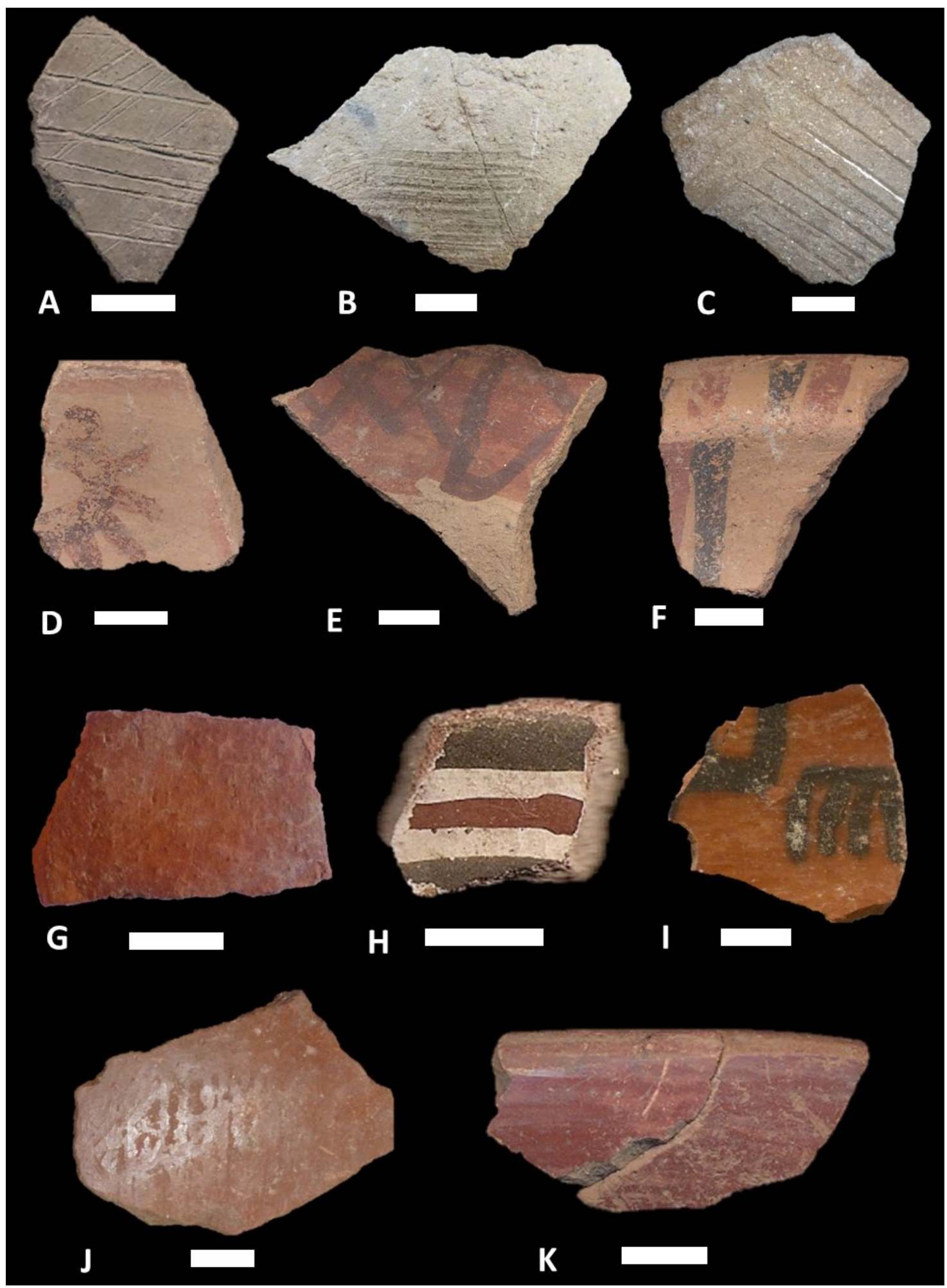

Figura 2. Tipos y conjuntos cerámicos definidos mencionados: A) Agrelo (Alberdi e Ituzaingó); B) Gris/Marrón (PA68); C) Gris/Marrón (PA19); D) escudilla Viluco Inca Mixto (Ruinas de San Francisco); E) jarra Viluco Inca Mixto (Alberdi e Ituzaingó); F) plato Viluco Colonial (Ruinas de San Francisco); G) cerámica Anaranjada Rojiza (PA93); H) cerámica Diaguita (Ruinas de San Francisco); I) tipo Copiapó Negro sobre Rojo (PA24); J) tipo Tocota (Rincón de Los Helados); K) Anaranjado pulido pintado de rojo (Rincón de los Helados).

Figure 2. Types and pottery assemblies: A) Agrelo (Alberdi e Ituzaingó); B) Gris/Marrón (PA68); C) Gris/Marrón (PA19); D) bowl Viluco Inca Mixto (Ruinas de San Francisco); E) jar Viluco Inca Mixto (Alberdi e Ituzaingó); F) plate Viluco Colonial (Ruinas de San Francisco); G) pottery Anaranjada Rojiza (PA93); H) pottery Diaguita (Ruinas de San Francisco); I) type Copiapó Negro sobre Rojo (PA24); J) type Tocota (Rincón de Los Helados); K) Anaranjado pulido pintado de rojo (Rincón de los Helados). 


\section{(Chiavazza y Prieto 2008).}

En el caso de la cerámica del período Alfarero Tardío y colonial temprano, el tipo diagnóstico más abundante y de mayor dispersión es Viluco (Figura 2 D, E), recuperado en sitios de la precordillera, piedemonte alto, piedemonte (valle de Mendoza) y planicie NE, incluyendo los sitios del río Desaguadero que son el límite oriental de su distribución (Prieto Olavarría et al. 2016) (Figura 1). Este tipo fue caracterizado en detalle, incluyendo los conjuntos fragmentarios y las vasijas enteras depositadas en colecciones (Prieto Olavarría 2012, Prieto y Chiavazza 2009). Su abundancia en los sitios del Área Fundacional (valle de Mendoza), permitió definir aspectos tipológicos, tecnológicos y decorativos de modo integral (Prieto Olavarría 2010a, 2012; Tobar 2013). La mayor parte de esta cerámica corresponde al tipo Viluco Inca Mixto (Ots 2008, Raffino 1981) y se caracteriza por la presencia de jarras, escudillas (con y sin apéndices), keros, aisana y aribaloides (Lagiglia 1978; Prieto Olavarría 2010a; 2010b; Prieto Olavarría 2012); las superficies son de tonalidades anaranjadas; la decoración pintada puede ser monocroma, bicroma o policroma con motivos geométricos; decoración modelada e incisa en jarras y escudillas con apéndices (Lagiglia 1978; Prieto Olavarría y Chiavazza 2009; Prieto Olavarría 2010a). Por otra parte, en el Área Fundacional de Mendoza se recuperaron platos Viluco que evidencian la integración de atributos Viluco (pasta y decoración) con otros de origen europeo (huella de uso de torno y forma de plato) (Prieto y Chiavazza 2009), por lo cual proponemos denominarlo Viluco Colonial (Figura 2 F).

A nivel petrográfico, el Viluco Inca Mixto arrojó cuatro grupos de fábrica (Granítico matriz micácea; Volcánico; Piroclástico; Granítico inclusiones arcillosas), destacando la predominancia de las inclusiones volcánicas y graníticas que se corresponden con la geología local (Prieto Olavarría y Castro de Machuca 2015). En dos casos fue posible definir preliminarmente las fuentes de origen de las inclusiones: las del grupo de fábrica granítico se corresponden con el granito del stock del Cerro Cacheuta; las piroclásticas podría corresponderse con los depósitos de la Planicie de Ignimbritas o Asociación Volcánica Piroclástica Pumícea (Prieto Olavarría y Castro de Machuca 2015). Esta última clase se inscribe dentro de la tradición de pastas con altos porcentajes de inclusiones piroclásticas, práctica que fue introducida durante la dominación incaica en las áreas más meridionales del imperio como el noroeste (NOA) y el centro oeste argentino (COA) (Prieto Olavarría y Páez 2015).

Las dataciones absolutas obtenidas de la cerámica Viluco la ubican entre mediados del siglo XV y mediados del XVII (Tabla 1), aunque un tiesto de la planicie arrojó una datación TL más temprana (780 180 años AP. UCTL 2699) y un fragmento de plato Viluco Colonial dio una fecha pre-colonial (530 \pm 50 años AP, UCTL 1971).
En Rincón de Los Helados (precordillera) y las grutas de Vaquería (piedemonte alto) también se recuperaron fragmentos de cerámica que tecno-tipológicamente se corresponden con los tipos producidos en los tambos incaicos de Uspallata (Chiavazza 1995, Chiavazza et al. 2003), concretamente los tipos Anaranjado pulido pintado de rojo (Figura 2 K) y Anaranjado pulido (Bárcena y Román 1990). Esto permitió conocer el área de dispersión de esta cerámica y definir aspectos relacionados con la movilidad durante la dominación incaica entre el valle de Uspallata y la precordillera (Chiavazza et al. 2003). El análisis petrográfico de un tiesto Anaranjado pulido pintado de rojo de Rincón de los Helados, posee un grupo de fábrica Volcánico granítico metamórfico, que si bien es diferente a los patrones Viluco comparte con él la abundante presencia de inclusiones volcánicas (Prieto Olavarría y Castro de Machuca 2015).

Un conjunto presente solamente en la planicie es el que denominamos preliminarmente como "Tardío" (Chiavazza 2007, Prieto Olavarría y Castro de Machuca 2015) y se caracteriza por las superficies alisadas o pulidas de tonalidades anaranjadas rojizas (Figura 2 $\mathrm{G}$ ) y decoraciones de pastillaje (tiestos con bandas onduladas con incisiones o impresiones identificados en Lagunas del Rosario) (Rusconi 1962). Se caracterizaron petrográficamente cuatro grupos de fábrica para este conjunto (Granítico matriz microgranosa; Granítico matriz micácea; Volcánica; Caliza) (Prieto Olavarría y Castro de Machuca 2015), y aunque la mayoría se corresponde con la geología del norte de Mendoza, el grupo Caliza (inclusiones micríticas, esparíticas y organógenas) debe ser evaluado en detalle debido a la presencia de microfósiles. Las dataciones absolutas permiten ubicarlo aproximadamente entre los ca. 600 y 800 años AP (Tabla 2 ), lo cual conduce a proponer que este conjunto alfarero fue producido en un período anterior a la cerámica Viluco Inca Mixto. Esta información es relevante, ya que hasta el momento se menciona que Agrelo es el tipo cerámico que antecede a Viluco (Canals Frau 1956). Debido a que este conjunto está en proceso de análisis, proponemos denominarlo grupo cerámico Anaranjado Rojizo.

Para el Alfarero Tardío también se recuperaron restos cerámicos de origen foráneo. El alero Rincón de Los Helados presenta conjuntos cerámicos poco abundantes pero diversos, entre los que se recuperaron restos de cerámica Tocota (Chiavazza 1995) (Figura 2 J), tipo descripto para el tambo homónimo de la provincia de San Juan (Berberián et al. 1981). Petrográficamente se observó que este tipo presenta gran cantidad de inclusiones piroclásticas, aunque abundan las trizas vítreas y no la pómez característica de Viluco (Prieto Olavarría y Páez 2015), por lo cual fue incluido dentro de las tradiciones de pastas con altos porcentajes de inclusiones piroclásticas características del NOA y COA (Prieto Olavarría y Páez 2015). Además, restos de cerámica Diaguita se recuperaron en diversos ambientes 
(piedemonte, piedemonte alto, Gruta 1 de Vaquería, planicie NE), los que se suman a la evidencia hallada en precordillera (Durán y García 1990, entre otros) (Figura 2 H). En la planicie también se hallaron dos fragmentos de un tortero confeccionado con cerámica del tipo Copiapó Negro sobre Rojo (Figura 2 I), asociado a un tiesto marrón rojizo bruñido sin adscripción tipológica y con una fecha entre la dominación inca y la colonia temprana $(445 \pm 40$ años AP. UCTL 2701) (Tabla 2).

\section{Discusión y conclusiones}

Los resultados obtenidos dan cuenta de la variabilidad cerámica existente en el norte de Mendoza y de su amplia distribución, especialmente de los tipos Agrelo y Viluco. Recientes estudios en el sector marginal del área norte sobre el río Desaguadero, confirman que ese fue el límite oriental de la dispersión de ambos conjuntos. Por su parte, las dataciones TL ubican a la cerámica Agrelo y la Gris/ Marrón dentro de un margen dado entre los ca. 1200 700 años AP y a la Viluco entre los ca. 700 - 450 años AP. En el caso de la cerámica del período Alfarero Medio, proponemos la necesidad de afinar las características tecno-tipológicas debido a la variabilidad observada.

Los datos indican que hay diversos tipos cerámicos representados en el Alfarero Tardío y la colonia temprana, y que la cerámica Viluco se extiende en toda el área de estudio hasta el río Desaguadero por el este. Además, los atributos tecnológicos de los platos hallados en el Área Fundacional permitieron definir un nuevo tipo, el Viluco Colonial, que se suma al tipo Viluco Inca Mixto producido desde la llegada de la expansión incaica al área. Esta evidencia permitirá evaluar la producción alfarera indígena durante la colonia en el marco de los cambios que se produjeron en los contextos de uso y consumo de las vasijas. Por otra parte, el reconocimiento de fragmentos de los tipos producidos en los tambos de Uspallata en los sitios de la precordillera y del piedemonte alto, posibilitó analizar la movilidad entre el valle de Uspallata y el piedemonte durante la dominación incaica. Respecto a la cerámica Anaranjada Rojiza registrada exclusivamente en la planicie $\mathrm{NE}$, cronológicamente se ubica en el período anterior a la cerámica Viluco Inca Mixto y planteamos que este conjunto, que es diferente tecnológicamente a las tradiciones del Alfarero Medio, habría sido la base de la tradición Viluco Inca Mixto.

Los análisis petrográficos permitieron caracterizar por primera vez el origen de las inclusiones no plásticas de cada tipo y definir que gran parte de los grupos de fábrica poseen inclusiones que se corresponden con la geología del norte de Mendoza. Las diferencias observadas entre los grupos de fábrica más abundantes de los tipos cerámicos del Alfarero Medio y Tardío, indican que en cada tradición cerámica se seleccionaron distintas fuentes de materias primas: en la cerámica Agrelo y Gris/ Marrón del período Medio abundan las inclusiones de origen sedimentario y menor cantidad las volcánicas y graníticas; en Viluco (Inca Mixto y Colonial) predominan los antiplásticos de origen volcánico o granítico y en menor proporción las sedimentarias.

Respecto a los tipos foráneos, la presencia de inclusiones características de las Sierras Centrales en un grupo de fragmentos del Alfarero Medio, permitirá seguir indagando en la movilidad e intercambio de las poblaciones del norte de Mendoza con aquellas ubicadas al norte y noreste de la provincia, hipótesis que se ha fortalecido con las recientes investigaciones desarrolladas en el río Desaguadero. Por su parte, para el Alfarero Tardío, la identificación de tipos no locales (Tocota, Diaguita y Copiapó Negro sobre Rojo) se relaciona directamente con la expansión incaica en el área.

El desarrollo de esta línea de trabajo, la inclusión de nuevos sitios y el re-análisis de algunos conjuntos de la planicie NE, permitirá ajustar aspectos tecno-tipológicos y cronológicos. La cerámica brinda evidencia relevante para continuar indagando sobre la movilidad y el intercambio entre los distintos ambientes del norte mendocino, con las áreas vecinas y sobre las transformaciones ocurridas en un lapso de casi 1000 años, especialmente las que se dieron luego del ingreso de incas y españoles.

\section{Bibliografía}

Bárcena J. \& A. Román. 1990. Funcionalidad diferencial de las estructuras del tambo de Tambillos: resultados de la excavación de los recintos 1 y 2 de la Unidad A del Sector III. Anales de Arqueología y Etnología 41-42: 7-81.

Bárcena, J. R. 1998. Arqueología de Mendoza. Las dataciones absolutas y sus alcances. EDIUNC, Serie Manuales. Mendoza.

Berberián, E., J. Martín de Zurita \& J. Gambetta. 1981. Investigaciones arqueológicas en el yacimiento incaico de Tocota (Prov. De san Juan, Rep. Argentina). Anales de Arqueología y Etnología 32-33: 173-218.

Boman, E. 1908. Antiquités de la région andine de la Républiqe Argentine et du desert d’Atacama Tomos I y II, París.

Boman, E. 1920. Cementerio Indígena de Viluco (Mendoza) Posterior a la Conquista. Anales del Museo Nacional de Historia Natural 30: 501-562.

Cahiza, P. 2001. Problemas y perspectivas en el estudio de la dominación Inca en las tierras bajas de Mendoza y San Juan: el sitio Torre 285, Retamito. Xama 12-13-14: 173-197.

Canals Frau, S. 1956. Algunos aspectos de la Cultura de Agrelo. Anales de Aqueología y Etnología Tomo XII: 7-18.

Canals Frau, S. \& J. Semper. 1956. La Cultura de Agrelo (Mendoza). Runa II (2): 69-180.

Castillo, L. 2007. Estudios cerámicos en el Noreste de la Provincia de Mendoza. Acondicionamiento y análisis del material cerámico recuperado en sitio PA70 Arroyo Tulumaya, Depto. de Lavalle, Mendoza. Informe SECyT, UNCuyo. Ms. 
Castillo L., F. Hernández, L. Mafferra, C. Frías, M. Quiroga, J. Anzorena \& A. Acosta. 2007. "Estudios Arqueológicos en el Sitio Tulumaya (PA 70): su inserción en el Patrón de Asentamiento Regional". En Actas del XVI Congreso Nacional de Arqueología Argentina, San Salvador de Jujuy, Jujuy. Pp.625-631.

Chiavazza, H. 1995. "Estudios Arqueológicos en el sitio "Rincón de Los Helados" ("RH"). Ocupación Multicomponente en Noreste de Pampa de Canota - Departamento de Las Heras, Provincia de Mendoza, República Argentina". Facultad de Filosofía y Letras, Universidad Nacional de Cuyo, Mendoza. Tesis de Licenciatura.

Chiavazza, H. 2001. Las antiguas poblaciones de las arenas. Arqueología de las Tierras áridas del Noreste mendocino. Serie Bienes Patrimoniales, Ediciones Culturales de Mendoza.

Chiavazza, H. 2007. "Cambios ambientales y sistemas de asentamiento en el árido normendocino. Arqueología en los paleocauces del río Mendoza". Facultad de Ciencias Naturales y Museo, Universidad Nacional de La Plata. Tesis para optar al título de Doctor en Ciencias Naturales. La Plata.

Chiavazza H. 2012. Ocupaciones humanas en la planicie árida Noreste de Mendoza: estudios arqueológicos en el Paleocauce Central (PC4). Relaciones Sociedad Argentina de Antropología XXXVII (2): 299-327.

Chiavazza, H. \& M. R. Prieto. 2008. Estudios arqueológicos en el Río Desaguadero (Mendoza). Runa, 29: 29-51.

Chiavazza H., V. Cortegoso \& L. Puebla. 2003. Sistemas de producción lítica en el piedemonte alto de la precordillera mendocina: el sitio Vaquería, Villavicencio. Anales de Arqueología y Etnología 54/55: 81-114.

Durán, V. y\& C. García. 1990. Ocupaciones Agroalfareras en el sitio Agua de la Cueva Sector Norte (NO de Mendoza). CEIDER Revista de Estudios Regionales 3: 29-64.

Frigolé, C., R. Moyano \& D. Winocur. 2014 Comparando la composición química y petrográfica de distintos estilos cerámicos en una casa del valle de Potrerillos (Mendoza, Argentina). En Arqueología de ambientes de altura de Mendoza y San Juan (Argentina). V. Cortegoso, V. Durán y A. Gasco (Eds), pp. 81-99. EDIUNC, Mendoza.

García, E. A. 1995. Nuevas perspectivas para el estudio de la cultura Agrelo. CEIDER Revista de Estudios Regionales 12: 19-27.

García, E. A. 1996. La dominación Inca en el centro oeste argentino y su relación con el origen y la cronología del registro arqueológico "Viluco". Anales de Arqueología y Etnología, 48/49: 41-48.

Lagiglia, H. 1978. La Cultura de Viluco del Centro Oeste Argentino. Revista del Museo de Historia Natural III (1-4): 227-265.

Michieli, C. 1974. "Arqueología de Mendoza en el período cerámico (entre los ríos Mendoza y Tunuyán". Facultad de Filosofía y Letras, Universidad Nacional de Cuyo. Seminario de Licenciatura en la carrera de Historia. Mendoza.

Ots, M. J. 2008. Aportes del Análisis Petrográfico de Cortes Delgados para la Caracterización y Clasificación del Estilo Cerámico Viluco Inka. Boletín del Laboratorio de Petrología y Conservación Cerámica Vol. 1: 12-20, №2, Año 1.

Prieto Olavarría, C. 2010a. La especialización artesanal alfarera de la cultura Viluco. Norte y centro de la provincia de Mendoza. Universidad Nacional de Córdoba Facultad de Filosofía y Humanidades. Tesis doctoral. Facultad de Filosofía y Humanidades, Universidad Nacional de Córdoba.

Prieto Olavarría, C. 2010b. Aproximaciones a la producción y función de la cerámica Viluco durante la dominación incaica y los primeros siglos de la Colonia en el valle de Mendoza. En J. R. Bárcena y H. Chiavazza (Eds.), Actas del XVII Congreso de Arqueología Argentina. Tomo I: 205-210. ZETA Editores, Mendoza.

Prieto Olavarría, C. 2012. La producción y función de la cerámica indígena durante la dominación incaica y la colonia en Mendoza (Argentina). Intersecciones en Antropología 13: 71-88.

Prieto Olavarría, C. \& H. Chiavazza. 2009. La producción cerámica Viluco entre los Siglos XV y XVII (Provincia de Mendoza, Argentina). Chungara, Revista de Antropología Chilena 41 (2), 261-274.

Prieto Olavarría, C. \& H. Chiavazza. 2010. La alfarería Viluco y los contextos del Área Fundacional. Aportes al estudio de la dominación incaica y los primeros años de la Colonia en el Valle de Mendoza, en: J. R. Bárcena y H. Chiavazza (Eds.), En Actas del XVII Congreso Nacional de Arqueología Argentina II: 807-812.

Prieto Olavarría, C. \& Castro de Machuca, B. 2015. Resultados preliminares del análisis petrográfico de la cerámica de los períodos agroalfarero medio y tardío del Norte de Mendoza. Xama Serie Monografías 5: 79-94.

Prieto Olavarría, C. \& Páez, C. 2015. Presencia de inclusiones piroclásticas en la cerámica de los siglos XV a XVII en el Centro Oeste y Noroeste Argentino. Chungara Revista de Antropología Chilena 47 (3), 441-453.

Prieto Olavarría, C., H. Chiavazza, L. Castillo, V. Porta \& E. Bontorno. 2015. Tecnología, tipología y cronología de la cerámica indígena del norte de Mendoza entre los ca. 20001-300 años ap. Ponencia presentada en las VI Jornadas de Arqueología Cuyana, Los Reyunos, San Rafael, octubre de 2015.

Prieto Olavarría, C., H. Chiavazza, V. Porta \& E. Bontorno. 2016. Estilos tecnológicos y procesos ambientales de la cerámica del río Desaguadero (PA68). Variabilidad cerámica en un sitio de límite cultural. Relaciones Sociedad Argentina de Antropología XLI (1): 131-150.

Raffino, R. 1981. Los Inkas del Kollasuyu. Origen naturaleza y transfiguraciones de la ocupación Inka en los Andes Meridionales. Ramos Americana Editora, Buenos Aires.

Rice, P. 1987. Pottery analysis: a source book. University of Chicago Press.

Rusconi, C. 1962. Poblaciones Pre y Post hispánicas de Mendoza Tomo II Arqueología. Edición oficial, Mendoza.

Tobar, V., 2013. "Los diseños decorativos de la cerámica Viluco (siglos XV-XVII): una aproximación a los lenguajes visuales locales". Facultad de Filosofía y Letras, Universidad Nacional de Cuyo. Tesina de licenciatura. Mendoza.

Whitbread, I. 1996. Detection and Interpretation of Preferred Orientation in ceramic thin section. Proceeding of the 2 Symposium of the Hellenic Archeometrical Society, pp. 413-425. 